\title{
Towards Health Informatics 3.0. Editorial
}

\author{
Casimir A. Kulikowski', Antoine Geissbuhler ${ }^{2}$ \\ 'Department of Computer Science, Rutgers - The State University of New Jersey, New Brunswick, NJ, USA \\ ${ }^{2}$ Department of Imaging and Medical Informatics, Geneva University Hospitals, Geneva
}

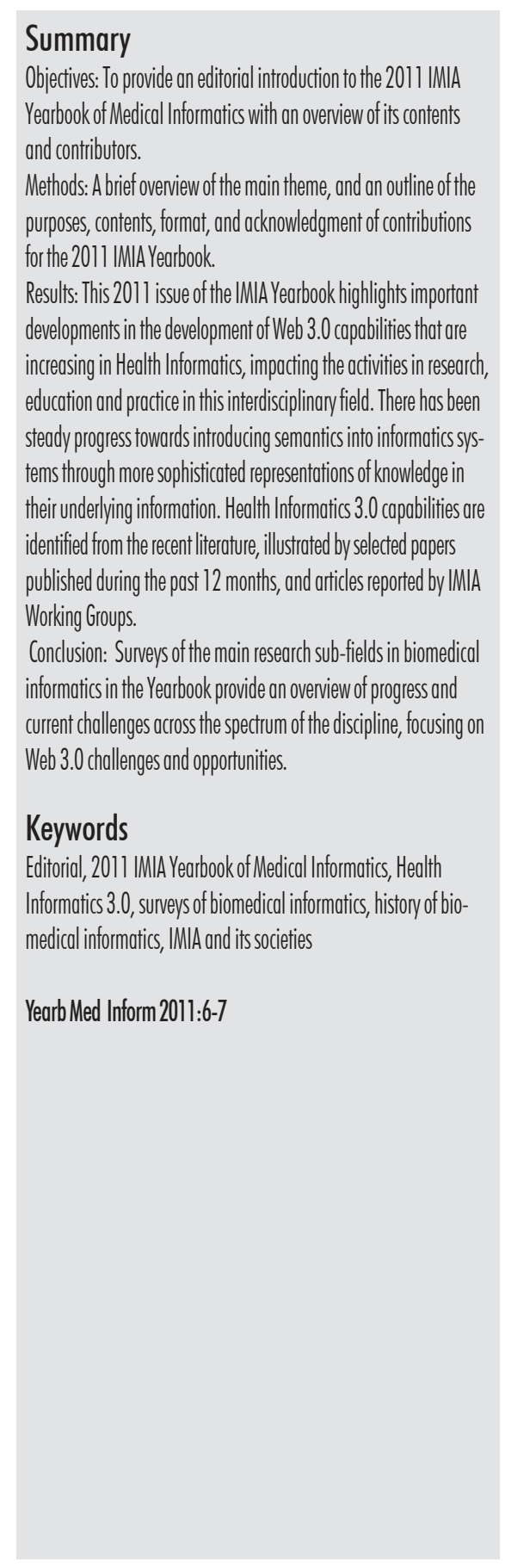

\section{Introducing the 2011 Yearbook and its Theme on Health Informatics 3.0}

The Yearbook includes a number of articles focusing on the theme of Health Informatics 3.0. It covers recent informatics contributions and points to future directions on how the gradual incorporation of semantic capabilities is enabling both more effective software inter-connectivity and more seamless, consistent ways of communication and collaboration among researchers and practitioners in Health Informatics over the web. The importance of this theme comes from the critical need to improve usability as well as portability of software, data, and methods for biomedical research, practice, and education. Increasing the effectiveness and dissemination of health informatics methods worldwide is a major goal of IMIA, and Web 3.0 approaches are increasingly central to its achievement. The Yearbook also reports on the successful World Congress of Medical Informatics (Medinfo 2010), held in Capetown, South Africa on September 13 of last year. This achievement emphasizes the increasing global reach and impact of health informatics, being the first Medinfo held in Africa. Its theme of Building Capacity Worldwide is especially relevant to help introduce advanced methods of informatics in regions where there are challenging health needs.

The 2011 IMIA Yearbook includes the following articles:

Keynote on the Theme of Health Informatics 3.0 - Dipak Kalra

Editorial, IMIA President Statement and IMIA Award Statement at Medinfo 2010.
Synopses and summaries of Best Paper Selections in each of the subfields of biomedical informatics.

Surveys in selected subfields of health informatics:

- Cheick Oumar Bagayoko (Health and Clinical Management)

- Petra Wilson (Sensors, Signals and Imaging Iformatics)

- Majid Altuwaijri (Consumer Informatics)

- Constantin Aliferis (Bioinformatics)

Working Group Articles:

- Thomas Karopka (WG Open Source Health Informatics)

- Chris Paton (WG Social Media)

- Alvin Marcelo (WG Health Informatics for Development)

- Paula Otero (WG Health and Medical Informatics Education)

- Michael Rigby (WG Technology Assessment \& Quality Development in Health Informatics)

- Anthony Maeder (WG Telehealth)

- Annie Lau (WG Consumer Health Informatics)

- Simon de Lusignan (WG Primary Health Care Informatics)

- Simon de Lusignan (WG Primary Health Care Informatics)

- Christian Lovis (WG Health Information Systems)

Research and Education:

- Rada Hussein: Biomedical and Health Informatics Education and Research at the Information Technology Institute in Egypt

History of Medical Informatics:

- Hans Petersen: Documenting the Use of Computers in Swedish Health Care up to 1980

- Jan van Bemmel: People and Ideas 
in Medical Informatics - a half century review

IMIA Award paper:

- Marion Ball: Back to the Future: What Have We Failed to Learn? How Does the Future Look?

Obituaries of International Leaders in Medical Informatics:

- Past President, IMIA - Professor Shigekoto Kaihara (Japan)

- Past President, Korean Institute of Medical Informatics (KOSMI) - Dr. Yun Sik Kwak (Korea)

- Co-Founder, European Society for Artificial Intelligence in Medicine, Professor Mario Stefanelli (Italy)

Report on the World Congress of Medical Informatics (Medinfo 2010) by Lyn Hanmer.

\section{About the Yearbook of Medical Informatics}

The Yearbook of Medical Informatics of the International Medical Informatics Association (IMIA) is distributed through IMIA's Member and Corresponding Member Societies worldwide. Since its inception in 1992 it has been the single most important publication summarizing the activities of IMIA, and showcasing the best of medical informatics research for the previous year. Due to changing demands by its readership and the expanded availability of original papers over the web, the IMIA Yearbook of Medical Informatics has adopted a new format and mode of publication since 2006, with the goal of substantially broadening its availability to virtually all members of the IMIA family. In particular, surveys, giving overviews of recent developments, and comprehensive introductory reviews on relevant topics in the different fields of health and biomedical informatics, have been added as original articles. The Yearbook remains a non-profit publication of IMIA, jointly published with Schattauer Verlag. It is currently subscribed to by 23 of IMIA's member societies, providing access for about 20.000 individual members.

\section{Information on IMIA and on its Regional Groups}

This past year IMIA successfully held its triennial conference MEDINFO, which, for the first time took place in Africa, in Cape Town in September 2010.

The Yearbook contains detailed information about IMIA, its Member Societies, Regional Groups, Working Groups, and Special Interest Groups.

A detailed report on the activities of IMIA regions is included with the help of Regional Editors. We would like to thank Rolf Engelbrecht (for EFMI), Michio Kimura (for APAMI), Ghislain Kouematchoua (for HELINA), Carol Hullin (for IMIA LAC), Ramin Moghaddam (for MEAHI) and Karen Greenwood and RoseMary MacVicarElliot (for the North American IMIA Member Societies) for their valuable contributions.

\section{Acknowledgements}

The editors gratefully acknowledge the contributions of the referees and guest editors. They would also like to thank the authors of the invited surveys, reviews and the historical paper, and the contributors to the Research and Education Section. They are most appreciative of the considerable skill, time, and effort devoted by Christian Lovis, the Managing Editor, and by the Section Editors, Claudine Bréant, Célia Boyer, Adrien Depeursinge, Arnaud Gaudinat, Alberto Guardia, Daniel Kubias, Rodolphe Meyer, Henning Müller, Anne-Marie Rassinoux, Patrick Ruch, and Lina Yip. They especially wish to thank the Editorial Assistant, Martina Hutter, from the Medical Faculty at the University of Heidelberg, without whose untiring efforts the Yearbook would not have been completed. The editors appreciate the contributions of the Advisory Board to the planning of this Yearbook. They are Reinhold Haux, Fernando Martin-Sanchez, Georges Mihalas, and Peter Murray.
The referees who contributed to the selection of articles in the 2011 Yearbook of Medical Informatics were:

Ameen Abu-Hanna, The Netherlands

Kouhei Akazawa, Japan

Fernan Gonzalez Bernaldo de Quiros, Argentina

Olivier Bodenreider, USA

Alex A.T. Bui, USA

Thomas Bürkle, Germany

Karen Courtney, USA

Dina Demner Fushman, USA

John T. Donnelly, USA

David Dorr, USA

Georg Duftschmid, Austria

Martin Dugas, Germany

HM Goh, Malaysia

Natalia Grabar, France

Stefan Gräber, Germany

Ghassan Hamarneh, Canada

Allan Hanbury, Austria

Heinz Handels, Germany

Dimitar Hristovski, Slovenia

Josef Ingenerf, Germany

Jayashree, Kalpathy-Cramer, USA

Jeongeun Kim, Korea

Mikko Korpela, Finland

Martin Krallinger, Spain

Pablo Laguna, Spain

Luigi Lo Iacono, Germany

Daniel Luna, Argentina

Shuqian Luo, China

Yasuhsi Matsumura, Japan

Stéphane Meystre, USA

Maryati Mohd Yusof, Malaysia

Jeannette Murphy, UK

Toshiharu Nakai, Japan

Jean Marc Palm, Canada

Natalia Pletneva, Switzerland

Daniel Racoceanu, Singapore

Dietrich Rebholz-Schuhmann, UK

Rainer Röhrig, Germany

Jacques Rougemont, Switzerland

Indra Neil Sarkar, USA

Michael Schumacher, Spain

Arash Shaban-Nejad, Canada

Maria-Ana Simonet, Switzerland

Lauge Sorensen, Denmark

Selma Supek, Croatia

Guy Tsafnat, Australia

Manolis Tsiknakis, Greece

Frank Ückert, Germany

Dimitri van de Ville, Switzerland

Ulrike Wittig, Germany

Toru Yao, Japan 